\title{
Homodyne Operation of a Phase-only Optical Amplifier
}

\author{
Joseph Kakande, Francesca Parmigiani, Radan Slavík, Periklis Petropoulos and David J. Richardson \\ Optoelectronics Research Centre, University of Southampton, Highfield Campus, Southampton, SO17 \\ 1BJ, UK, jkk@soton.ac.uk
}

\begin{abstract}
We utilise cascaded four wave mixing to multiply the modulation depth of a phase-only optical signal and generate a comb of phase locked local oscillators allowing homodyne retrieval of the phase information with enhanced fidelity.
\end{abstract}

\section{Introduction}

Analogue photonic signal processing is gaining its momentum in several fields traditionally dominated by electronics, including analogue-todigital conversion $(A D C)^{1}$, microwave and terahertz-wave generation ${ }^{2,3}$, radio-over-fiber $(\mathrm{ROF})^{4}$ and all-optical regeneration ${ }^{5,6}$. The reasons for this migration are well known, centered around the large bandwidth*distance products achievable, as well as the greater measurement fidelity attainable when performing functions such as sampling alloptically ${ }^{1}$. In these analogue systems, certain functions are required to groom the parameters of the optical signal, the most common being amplification. The use of optical amplifiers allows one to boost the signal power above the eventual optoelectronic receiver's noise floor, and thus, to maximize the detected signal-tonoise ratio (SNR). Recently, we proposed the optical phase-only amplifier as a means of processing phase encoded optical signals prior to the optoelectronic receiver. In contrast to the conventional optical amplifier, in which the optical signal and information-bearing sidebands are amplified equally, in the phase-only amplifier the sidebands are enhanced without boosting the redundant carrier, dramatically improving the received SNR. The technique works by using cascaded four wave mixing (FWM) to generate a comb of harmonics from the modulated signal and a local continuous wave (CW) pump, and as one observes the resulting harmonics extending away from the signal, each one possesses a perfectly linear increase in modulation depth (relative to the preceding one). In simple terms, if the input signal possesses phase modulation of depth $\varphi$, the $\mathrm{n}^{\text {th }}$ harmonic bears modulation depth $n \varphi$. In the weak modulation regime, this can boost the signal power above the noise floor of the optoelectronic receiver, and we have previously used this to demonstrate close to 3 extra effective number of bits (ENOB) in the digitization process of an analogue signal by detecting the $8^{\text {th }}$ harmonic for an input signal modulated weakly in phase at $32 \mathrm{GHz}^{\text {? }}$.

One potential concern regarding our implementation of the phase-only amplifier was that the phase-multiplied signal was detected differentially, in a delay-line interferometer (DLI). For some of the applications that we envisage, such as optically assisted ADC, it is important to detect the signal coherently, so that the distortive effect of differential detection (a DLI has a frequency dependent response) is not perceived. It is therefore important to generate a comb of local oscillators, locked in relative phase to the signal and its phase-multiplied harmonics. Here, we propose a method of achieving this, and verify that this concept

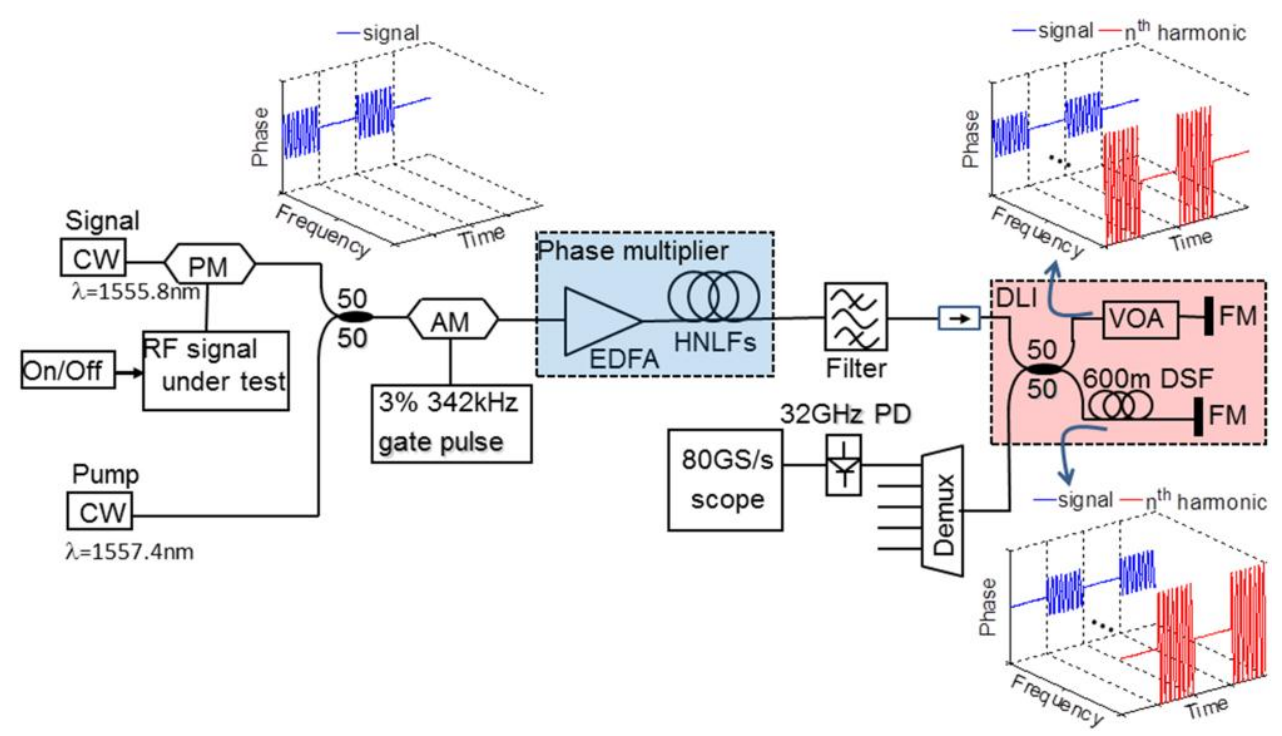

Fig. 1: Phase amplification experimental set-up. PM: phase modulator, AM: Amplitude modulator, VOA: variable optical attenuator, FM: Faraday mirror, DSF: dispersion shifted fiber. 
operates successfully.

\section{Operation Principle and Experimental set-up}

The optical signal,

is mixed with a CW pump,

$$
\exp \left[i\left(2 \pi f_{s} t+\varphi_{s}(t)\right)\right]
$$

$$
\exp \left[i\left(2 \pi\left(f_{s}+\Delta f\right) t+\varphi_{p}\right)\right]
$$

where $f_{s}$ is the signal carrier frequency, $\Delta f$ the frequency separation between pump and signal, $\varphi_{s}(t)$ the fast time-varying signal phase (encoded information) and $\varphi_{p}$ the slowly varying phase difference between pump and signal (comparable to their underlying linewidths). Following mixing, a comb of harmonics is generated (Fig 2) where the nth harmonic (neglecting its power) can be written as ${ }^{7}$

$$
\begin{aligned}
\exp \left[i \left(2 \pi\left(f_{s}-(n-1) \Delta f\right) t\right.\right. & \\
& \left.\left.+n \varphi_{s}(t)-(n-1) \varphi_{p}\right)\right]
\end{aligned}
$$

and the $n \varphi_{s}(t)$ term shows the phase multiplication. Note that $\mathrm{n}=1$ corresponds to the original signal. To demodulate the nth harmonic in a homodyne fashion, a local oscillator must be provided at frequency $f_{s}-(n-1) \Delta f$, locked to the slowly varying $(n-1) \varphi_{p}$ phase term on the harmonic, the harmonic's phase reference. While one could potentially envisage doing so for a single harmonic using a free running local oscillator controlled by an optical feedback loop, it is preferable to have a local oscillator comb present tracking all $n$ harmonics simultaneously. Our solution is quite straightforward. We propose to have a second FWM comb in which a modulation-free copy of the signal (i.e. the signal carrier) is mixed with the same local pump. In this case, the nth harmonic of this second comb can be written as

$$
\exp \left[i\left(2 \pi\left(f_{s}-(n-1) \Delta f\right) t-(n-1) \varphi_{p}\right)\right]
$$

and therefore by beating this second FWM comb with the first, all the $\mathrm{n}$ harmonics can simultaneously be demodulated in a homodyne manner. There are a number of techniques in literature to recover the carrier from a modulated optical signal ${ }^{8}$. However, to verify the concept, and assess its performance irrespective of what carrier recovery scheme is eventually chosen, we generated our two combs as follows.

While in future implementations we intend to have two separate four wave mixing stages, one for 'modulated signal + pump' and the other for 'signal carrier + pump', we chose to use a single optical fiber, and simply turn the signal phase modulation on and off at $171 \mathrm{kHz}$ (frequency explained later). The result of this is that for half of this $171 \mathrm{kHz}$ cycle we have access to the modulated signal, and for the rest of the time to its carrier. By mixing this hybrid optical wave with the local pump in a low-dispersion highly nonlinear fiber (HNLF), we obtain our comb of
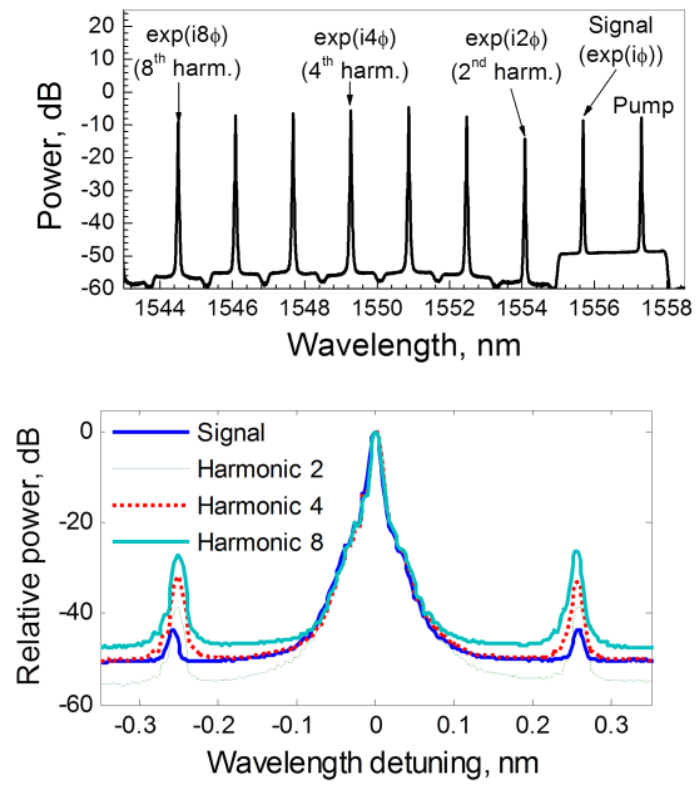

Fig. 2: Top) HNLF output spectra, CW operation. Bottom) Overlaid optical spectra of harmonics 1 (signal), 2, 4 and 8 after the HNLFs when the signal was phase modulated at $32 \mathrm{GHz}$.

cascaded FWM harmonics. It follows that for half of a $171 \mathrm{kHz}$ cycle this FWM is of 'modulated signal + pump', and for the other half of 'signal carrier + pump'. To perform signal demodulation, we simply need to overlap in time these two time-separated combs, which we do using a Michelson interferometer with a delay of $600 \mathrm{~m}$. It must be emphasized that this solution is only interim, and in future demonstrations the two combs will be spatially separate, and not temporally as in this case.

The experimental set-up is shown in Fig.1. The signal information is modulated onto an optical carrier at $1555.8 \mathrm{~nm}$ using a $40-\mathrm{GHz} \mathrm{LiNbO}_{3}$ phase modulator. The signal was then combined with a CW pump at $1557.4 \mathrm{~nm}$. The signal and pump were gated at a repetition rate of $342 \mathrm{kHz}$ and $3 \%$ duty cycle to boost the peak power by a factor of $15 \mathrm{~dB}$, while avoiding stimulated Brillouin scattering (SBS) in the HNLF, properly filtered and amplified to a total average power in the HNLF up to $100 \mathrm{~mW}$. The earlier selected value of $171 \mathrm{kHz}$ to gate the RF signal now becomes clear; it is a sub-multiple of the 342 $\mathrm{kHz}$ (arbitrary choice) gating frequency. The nonlinear optical section consisted of two HNLFs connected by $12 \mathrm{~m}$ of SMF-28. The length, dispersion, dispersion slope, nonlinear coefficient and attenuation of the HNLFs at 1550 $\mathrm{nm}$ were as follows: $500 \mathrm{~m}, 0.06 \mathrm{ps} / \mathrm{nm} / \mathrm{km}$, $0.0035 \mathrm{ps} / \mathrm{nm}^{2} / \mathrm{km}, 18 \mathrm{~W}^{-1} \mathrm{~km}^{-1}$ and $1.4 \mathrm{~dB} / \mathrm{km}$ and $500 \mathrm{~m},-0.09 \mathrm{ps} / \mathrm{nm} / \mathrm{km}, 0.016 \mathrm{ps} / \mathrm{nm}^{2} / \mathrm{km}$, $11.5 \mathrm{~W}^{-1} \mathrm{~km}^{-1}$ and $0.8 \mathrm{~dB} / \mathrm{km}$, respectively. Fig. 2 (top) shows the optical spectrum of the FWM comb with harmonics up to $n=8$ visible (with 


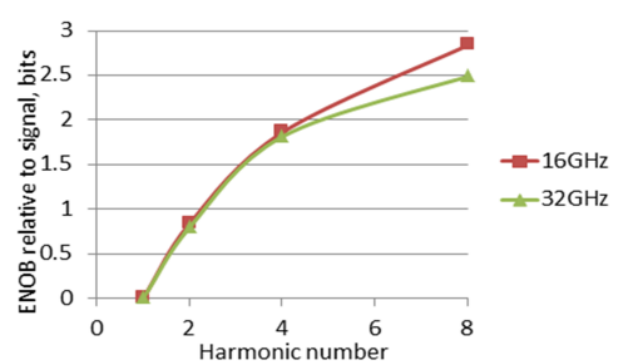

Fig. 4: ENOB of various harmonics at 16 and 32 $\mathrm{GHz}$ as calculated from the detected RF data in the $0-40 \mathrm{GHz}$ range.

the RF signal off). A difference of a few $\mathrm{dB}$ between the first and $8^{\text {th }}$ harmonic is feasible without significant optical signal-to-noise ratio (OSNR) degradation. Following the Michelson interferometer, in which we interfere the 'modulated signal + pump' and 'signal carrier + pump' combs, the now homodyne-demodulated harmonic channels were individually selected using a bandpass filter and characterized using a $32-\mathrm{GHz}$ photodetector and an $80 \mathrm{GS} / \mathrm{s}$ real time oscilloscope with a $32 \mathrm{GHz}$ bandwidth. Note that the maximum possible signal power was coupled into the detector (as per the detector's specification) to achieve optimal optical-to-electrical conversion.

\section{Results}

Fig. 3 (bottom) shows the overlaid optical spectral traces of the original signal, $2^{\text {nd }}, 4^{\text {th }}$ and $8^{\text {th }}$ harmonics when a $32 \mathrm{GHz}$ sine wave is imposed on the signal. The applied peak-topeak voltage was about $0.01 \mathrm{~V}_{\pi}$. The $8^{\text {th }}$ harmonic is increased in power by about $16 \mathrm{~dB}$, with the sideband OSNR improved by about 13 $\mathrm{dB}$. The analog detector output was captured with the real time oscilloscope and processed offline to calculate standardized figures of merit - such as signal to noise and distortion ratio (SINAD) and ENOB, for a frequency range of 1$40 \mathrm{GHz}^{9}$. An increase of ENOB of almost 3 bits (Fig.4) and SINAD improvement of about $17 \mathrm{~dB}$ is observed from the $1^{\text {st }}$ to the $8^{\text {th }}$ harmonic for signal modulation at $16 \mathrm{GHz}$.

Finally, to show the benefit of performing homodyne, rather than differential demodulation, the signal was phase encoded with a wideband input RF test pattern, occupying $10 \mathrm{GHz}$, and generated using a 24 GS/s Arbitrary Waveform Generator (AWG). The raw electrical pattern can be seen in Fig. 5 (top). The corresponding harmonics (1, 2, 4 and 8) after detection and normalisation are shown in Fig. 5. As expected, the higher the harmonic detected, the less the amount of noise visible. Also, because the detection method is homodyne rather than differential, the detected electrical signal closely

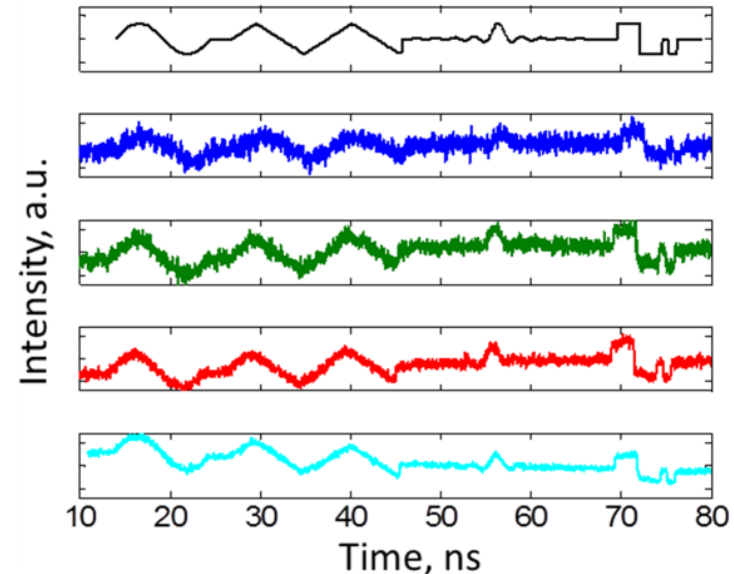

Fig. 5: Normalized RF test phase pattern and signal, harmonic 2, 4 and 8 after demodulation

(from top to bottom respectively).

resembles the applied test pattern, in contrast to the result in our previous work ${ }^{7}$.

\section{Conclusions}

We have used an optical phase-only amplifier to enhance the modulation depth of a phase encoded signal prior to demodulation and detection, leading to almost 3 extra effective number of bits in detection. The detection was performed in a homodyne fashion by generating a local oscillator comb serving as a phase reference for the phase multiplied optical harmonics. This homodyne detection opens up a route to using the device as a building block in optically assisted ADCs in future.

\section{Acknowledgements}

FP gratefully acknowledges the support from the Royal Academy of Engineering/EPSRC through a University Research Fellowship. This work is supported by the EPSRC grant EP/I01196X: The Photonics Hyperhighway.

\section{References}

[1] A. Khilo et al, Opt. Express 20, 4454-4469 (2012).

[2] A.J. Seeds and K.J. Williams, J. Lightwave Technol. 24, 4628-4641 (2006).

[3] S. Fukushima, et al., J. Lightwave. Technol. 21, 3043-3051 (2003).

[4] Y. Kim, et al., Opt. Express 16, 1068-1076 (2008).

[5] K. Croussore, et al., Opt. Express 14, 20852094 (2006).617-619 (2003).

[6] R. Slavík et al. Nat. Photonics 4(10), 690695 (2010).

[7] J. Kakande et al., OFC 2012, PDP 5C.9.

[8] R. Slavík, et al. Opt. Express 19, 2659426599 (2011).

[9] "IEEE Standard for Terminology and Test Methods for Analog-To-Digital Converters," IEEE Std 1241-2000, vol., ( 2001). 